\title{
The Medical Management of Arsha with Chirabilwadi Quatha and Kaseesadi Thaila Sthanika Abhyanga
}

\author{
Research Article
}

\section{Nagesh Gandagi $^{*}$, Shubhangi Patil ${ }^{2}$}

1, Reader \& Physician, Dept. of Kayachikitsa, 2, H.O.D. \& Reader, Dept. of Roganidan, Ahalia Ayurveda Medical College \& Hospital, Palakkad, Kerala

\begin{abstract}
Arsha (Piles) is the one of the most common disease encountered in clinical and surgical practice. Arsha incidence increases with advancing age, at least $50 \%$ of people over the age of 50 years have some degree of haemorrhoidal symptoms. In this study an attempt has been made to treat the "disease" with Chirabilwadi quatha (Panartha) and Kaseesadi thaila (Sthanika abhyanga). Total thirty patients were selected by simple randomized method and allocated into three groups called Group A, B, C. Each group had ten patients. Group A. treated with Chirabilwadi quatha (Panartha), Group B. treated with Kaseesadi thaila and Group C. treated with combination of Chirabilwadi quatha and Kaseesadi thaila. Total study period was nighty days in that thirty days was treatment and sixty days was follow-up. The effect of treatment was observed both in subjective symptoms and objective parameters. Nonsignificant, Significant and highly significant results were observed in subjective parameters, objective parameters and overall in all three groups.
\end{abstract}

Key words: Arsha, Piles, Chirabilwadi quatha, Kaseesadi thaila.

\section{Introduction}

Arsha is a kind of disease which is most unkind towards mankind. By looking into the history one can understand that great saints like adishankara who revived the vedic literature, and famous rulers like Napoleon suffered from this disease (1). Arsha incidence increases with advancing age, at least $50 \%$ of people over the age of 50 years have some degree of haemorrhoidal symptoms (2). Now a day every person suffered from any one of the complaint of piles during their life time.

*Corresponding Author:

Nagesh Gandagi

Reader, Department of Kayachikitsa

Ahaliya Ayurveda Medical College \&

Hospital

Palakkad, Kerala

E-mail: drnagasvgm@ rediffmail.com

Mobile No: +91-9986319930
Causes may be due to portal hypertension, hereditary or congenital, constipation, prostatic enlargement, pregnancies, long standing postures, insufficient dietic fiber (3). etc... As per Sushruta the doshas by their aggravating causes, dislodges from their normal seats alone or combined with other including the rakta, reaches the maladwara (marga) and causes the vitiation of gudavalis resulting the production of mamsankuras especially in mandagni persons ( 4 ). In this present era more number of patients are interested or inclined to take oral medicines rather than surgical procedures because they knew that surgical procedures may give raise one or other complications (5). Hence, aimed to study the first line of treatment of Sushrutha which is known as oushadha chikitsa (4). Good results of initial clinical trials of chirabilwadi quatha (6,8) as panartha and kaseesadi thaila $(7,8)$ as sthanika abhyanga 
inspired me to take up the present work. "The medical management of arsha with chirabilwadi quatha and kaseesadi thaila sthanika abhyanga".

Aim:

1. This study was conducted to evaluate the efficacy and safety of Chirabilwadi quatha and Kaseesadi thaila in Arsha.

\section{Objectives}

1. Primary efficacy objective - Study of the effect of Chirabilwadi quatha and Kaseesadi thaila on changes in parameters - pain, bleeding, nature of stool, pressure on defecation, pruritus ani, mucous discharge, number of external and internal pile mass, size, character, tenderness on pressure, movement, from Baseline to after treatment in Arsha (Piles).

2. Secondary efficacy objective - Study of the effect of Chirabilwadi quatha and Kaseesadi thaila on changes in parameters - pain, bleeding, nature of stool, pressure on defecation, pruritus ani, mucous discharge, number of external and internal pile mass, size, character, tenderness on pressure, movement, from Baseline to after Follow up in Arsha (Piles).

3. Exploratory efficacy objective - Study of the effect of Chirabilwadi quatha and Kaseesadi thaila on changes in parameters - pain, bleeding, nature of stool, pressure on defecation, pruritus ani, mucous discharge, number of external and internal pile mass, size, character, tenderness on pressure, movement, from Baseline to After treatment (30 days, After Follow up (90 days) in Arsha (Piles).

4. Safety objective - Evaluation of the safety of Chirabilwadi quatha and Kaseesadi thaila in term of the occurrence of adverse events, changes from baseline in vital signs (B.P. Heart and Respiratory rate) and safety laboratory results (Bio-chemistry, Hematology ) in Arsha (Piles) patients.

\section{Hypothesis}

Recent statistics reveals that irrespective of age, sex, socio - economic status, people suffer from piles. Now a day every person suffered from any one of the complaint of piles during their life time. Arsha incidence increases with advancing age, at least $50 \%$ of people over the age of 50 years have some degree of haemorrhoidal symptoms. The food habits and life styles of modern man also added to the increase in rate of incidence of Arshas. Piles pierce through may appear as a simple haemorrhoidal masses that descending.

1. Component of Hypothesis which to be total tested - Is there any presence of a positive effect relationship on the Arsha (Piles) patients who taken Chirabilwadi quatha and Kaseesadi thaila.

2. Component could not be tested Assessment of the G.I.Tract, kidney and liver parameters because Chirabilwadi quatha and Kaseesadi thaila is helpful in the long term use.

3. Comments over hypothesis testing Symptoms like pain, bleeding, Nature of stool, pressure on defecation, pruritus ani, mucous discharge and other studied but more importance given to pain of Arsha (Piles). As it interferes the day to day activities. That's why more emphasis is given on symptomatic relief. Before treatment, After treatment and After Follow-up is to be tested so that one may observe deterioration, stable, improvement and marked respond.

4. This Hypothesis was subjected to testing by using open trial clinical study.

5. This proposed operative Hypothesis for the present study is derived on the basis of

A. The description in the classical Ayurvedic texts. 
B. The clinical experiences of doctors in contemporary medicine and as well as in classics.

C. The survey of the literature regarding contemporary research on Arsha (Piles).

\section{Materials:}

Following were the materials taken for the clinical study.

\section{Drugs:}

1. Chirabilwadi Quatha

2. Kaseesadi thaila

\section{Instruments:}

1. Proctoscopy (Lighted)

2. Lithotomy Table

3. Stainless steel spoon (modified)

4. Hand gloves

5. Cotton ball

Methods:

Study design

The study was an open clinical trial conducted in Dr. B.N.M.E.T's Shri Mallikarjun Swamji's Post Graduate and Research Center, Bijapur. A total number of thirty patients were selected incidentally on the basis of inclusion criteria. The patients thus selected were randomly allocated to Three groups assigned as A, B and $\mathrm{C}$ were treated with chirabiwadi quatha, kaseesadi thaila and chirabilwadi quatha followed by kaseesadi thaila respectively.

GROUP - A. $=$ In this group the patients were administered $10 \mathrm{ml}$ of chirabilwadi quatha with sukhoshna jala, paschat bhakta, prathaha and ratrikala for 1 masa followed by takra as pathya.

GROUP - B. = In this group the patients were get sthanika abhyanga on Arsha with kaseesadi taila, santulita matra, paschat malapravruthi for 1 masa followed by takra as pathya.
GROUP $-\mathrm{C} .=$ In this group the patients were followed group $-\mathrm{A}$ and group $-\mathrm{B}$ as a combined.

The patients were advised to attain the O.P.D. for periodic follow-up. They were also advised to consult immediately, if any discomfort is felt in the course of follow-up period. Total 90 days trial period in that 30 days treatment and 60 days follow up period. Data is recorded before treatment, after treatment and after follow up on subjective and objective criteria and entered in a special clinical proforma which was prepared with relevance to the Ayurvedic and Allied science. A written informed consent was obtained from all patients.

Sample size - A total of thirty patients were selected incidentally on the basis of prevalence of Arsha (Piles) quoted in the text one can calculate the minimum required sample size for the study by using marginal error or standard error. The patients thus selected were randomly allocated to three groups.

\section{Inclusion Criteria}

1. Arsha diagnosed according to classical and modern signs and symptoms and on examinations.

2. Patients were selected irrespective of sex, religion and place.

3. Patients were selected in between 20 to 50 years age group and of any degree any type of Arshas.

\section{Exclusion Criteria}

1. Age below 20 years and above 50 years.

2. Associated with fistula and polyp.

3. Carcinoma of rectum, prolapsed rectum, congenital abnormalities of anus.

4. Gangrene, Fibrosis Suppuration of piles.

5. Any systemic disorders as Anaemia etc.

6. Debilitating disorders as Tuberculosis, Rheumatoid arthritis, oncology etc. 


\section{Pilot Study}

A pilot study on 6 patients was done, in order to grade the severity for individual symptom and over all severity dose fixation, determine end point and grading for the assessment of clinical improvement.

\section{Investigations}

$\mathrm{Hb} \%$, R.B.S., Urine routine etc (If found necessary)

\section{Assessment of Variables}

Clinical assessment was made for the severity of the disease and for the clinical improvement.

Grading for the severity of individual subjective and objective parameters, assessment and as well as for overall assessment was framed as four point scale (1-4). The gradings of 12 variables is given along with clinical proforma especially designed for the study on Arsha.

The severity of each variable ranging from Normal - 1, Mild - 2, Moderate - 3, Severe -4 .

\section{Overall Assessment of Severity}

Before treatment (Starting day), After treatment (after 30 days), After follow-up (after 90 days). Based on the scoring of variables, overall severity was graded as

Prakrita (Normal): 1 to 12, Mrudu (Mild): 13 to 24, Madyama (Moderate): 25 to 36 and Teevra (Severe): 37 to 48.

\section{Assessment of Clinical Improvement}

Clinical improvement of the disease was based on improvement in the clinical findings and reduction in the severity of the symptoms and overall severity of the disease. Grading for the clinical improvement individual symptoms and over all severity is as enumerated below.
Grading For the Clinical Improvement For Individual Variable

C.D. - Clinically deteriorated, i.e. increase in severity score against the initial score.

1) C.S. - Clinically stable, i.e. severity score remains as against the initial score.

2) C.I. - 1 - Encouraging i.e. 1 degree reduction in the severity score, against the initial score. i.e. reduction from mild - normal, moderate - mild, severe - moderate.

3) C.I. -2 -Good i.e. 2 degree reduction in the severity score, against the initial score. i.e. reduction from moderate - normal, sever - mild.

4) C.I. -3 -Excellent i.e. 3 degree reduction in the severity score, against the initial score. i.e. sever normal.

Grading for the clinical improvement on over all severity.

1) C.D: Deteriorate (increase in the severity score)

2) C.S: Stabilized (no change in the severity score)

3) C.I-1: Encouraging (1-12 reduction in the severity score)

4) C.I-2: Good (13-24 reduction in the severity score)

5) C.I - 3: Excellent (25-36 reduction in the severity score)

\section{Data Collection}

Assessment for individual signs and symptoms and overall clinical improvement was done. The data was collected from each group at Before treatment, After treatment and After follow-up. Scoring was done and finally the data was collected, compared and analyzed, tabulated as convenient.

\section{Statistical Analysis}

The data collected were statistically analyzed with the help of microsoft excel ${ }^{\mathrm{XP}}$ version under the guidance of statistician. 
The data was computed for mean standard deviation, standard error, ' $\mathrm{t}$ ' value and $\mathrm{P}$ Values. $P$ value was obtained using student's paired ' $t$ ' test. Significance of the results was based on the $\mathrm{P}$ value. The statistical values from each group were collected and tabulated as convenient or compared and analyzed.

RESULT RELATED OBSERVATIONS FOR INDIVIDUAL GROUPS:

CHARTS OF RESPONSE AT THE END OF TREATMENT

\begin{tabular}{|c|c|c|c|c|c|c|}
\hline \multicolumn{7}{|c|}{ RESPONSE OF THE THERAPIES FOR INDIVIDUAL } \\
\hline GROUPS & & ERC & AGE) & R TRI & IENT & \\
\hline Variables & Groups & CD & CS & C1-1 & C1-2 & C1-3 \\
\hline \multirow{3}{*}{ Pain } & $\mathrm{A}$ & $0 \%$ & $70 \%$ & $10 \%$ & $20 \%$ & $0 \%$ \\
\hline & $\mathrm{B}$ & $0 \%$ & $0 \%$ & $30 \%$ & $50 \%$ & $20 \%$ \\
\hline & $\mathrm{C}$ & $0 \%$ & $0 \%$ & $10 \%$ & $60 \%$ & $30 \%$ \\
\hline \multirow{3}{*}{ Bleeding } & A & $0 \%$ & $10 \%$ & $40 \%$ & $50 \%$ & $0 \%$ \\
\hline & B & $0 \%$ & $0 \%$ & $50 \%$ & $40 \%$ & $10 \%$ \\
\hline & $\mathrm{C}$ & $0 \%$ & $80 \%$ & $0 \%$ & $10 \%$ & $10 \%$ \\
\hline \multirow{3}{*}{ Nature of Stool } & A & $0 \%$ & $20 \%$ & $40 \%$ & $40 \%$ & $0 \%$ \\
\hline & $\mathrm{B}$ & $0 \%$ & $90 \%$ & $10 \%$ & $0 \%$ & $0 \%$ \\
\hline & $\mathrm{C}$ & $0 \%$ & $0 \%$ & $10 \%$ & $60 \%$ & $30 \%$ \\
\hline \multirow{3}{*}{ Pressure on defecation } & $\mathrm{A}$ & $0 \%$ & $0 \%$ & $20 \%$ & $40 \%$ & $40 \%$ \\
\hline & $\mathrm{B}$ & $0 \%$ & $90 \%$ & $10 \%$ & $0 \%$ & $0 \%$ \\
\hline & $\mathrm{C}$ & $0 \%$ & $0 \%$ & $10 \%$ & $60 \%$ & $30 \%$ \\
\hline \multirow{3}{*}{ Pruritus ani } & A & $0 \%$ & $0 \%$ & $30 \%$ & $60 \%$ & $10 \%$ \\
\hline & B & $0 \%$ & $0 \%$ & $60 \%$ & $40 \%$ & $0 \%$ \\
\hline & $\mathrm{C}$ & $0 \%$ & $0 \%$ & $30 \%$ & $40 \%$ & $30 \%$ \\
\hline \multirow{3}{*}{ Mucous Discharge } & $\mathrm{A}$ & $0 \%$ & $0 \%$ & $20 \%$ & $70 \%$ & $10 \%$ \\
\hline & B & $0 \%$ & $0 \%$ & $60 \%$ & $40 \%$ & $0 \%$ \\
\hline & $\mathrm{C}$ & $0 \%$ & $0 \%$ & $30 \%$ & $40 \%$ & $30 \%$ \\
\hline \multirow{3}{*}{ External } & A & $0 \%$ & $100 \%$ & $0 \%$ & $0 \%$ & $0 \%$ \\
\hline & B & $0 \%$ & $100 \%$ & $0 \%$ & $0 \%$ & $0 \%$ \\
\hline & $\mathrm{C}$ & $0 \%$ & $100 \%$ & $0 \%$ & $0 \%$ & $0 \%$ \\
\hline \multirow{3}{*}{ Internal } & $\mathrm{A}$ & $0 \%$ & $100 \%$ & $0 \%$ & $0 \%$ & $0 \%$ \\
\hline & $\mathrm{B}$ & $0 \%$ & $100 \%$ & $0 \%$ & $0 \%$ & $0 \%$ \\
\hline & $\mathrm{C}$ & $0 \%$ & $100 \%$ & $0 \%$ & $0 \%$ & $0 \%$ \\
\hline \multirow{3}{*}{ Size } & A & $0 \%$ & $30 \%$ & $30 \%$ & $40 \%$ & $0 \%$ \\
\hline & B & $0 \%$ & $0 \%$ & $40 \%$ & $50 \%$ & $0 \%$ \\
\hline & $\mathrm{C}$ & $0 \%$ & $0 \%$ & $0 \%$ & $60 \%$ & $40 \%$ \\
\hline \multirow{3}{*}{ Character } & A & $0 \%$ & $30 \%$ & $30 \%$ & $40 \%$ & $0 \%$ \\
\hline & B & $0 \%$ & $10 \%$ & $40 \%$ & $50 \%$ & $0 \%$ \\
\hline & $\mathrm{C}$ & $0 \%$ & $0 \%$ & $0 \%$ & $70 \%$ & $30 \%$ \\
\hline \multirow{3}{*}{ Pressure on tenderness } & A & $0 \%$ & $70 \%$ & $0 \%$ & $30 \%$ & $0 \%$ \\
\hline & $\mathrm{B}$ & $0 \%$ & $0 \%$ & $60 \%$ & $40 \%$ & $0 \%$ \\
\hline & $\mathrm{C}$ & $0 \%$ & $0 \%$ & $50 \%$ & $50 \%$ & $0 \%$ \\
\hline \multirow{3}{*}{ Movement } & A & $0 \%$ & $60 \%$ & $0 \%$ & $30 \%$ & $10 \%$ \\
\hline & $\mathrm{B}$ & $0 \%$ & $90 \%$ & $0 \%$ & $10 \%$ & $0 \%$ \\
\hline & $\mathrm{C}$ & $0 \%$ & $90 \%$ & $0 \%$ & $10 \%$ & $0 \%$ \\
\hline \multirow{3}{*}{ Over all } & $\mathrm{A}$ & $0 \%$ & $0 \%$ & $100 \%$ & $0 \%$ & $0 \%$ \\
\hline & B & $0 \%$ & $0 \%$ & $100 \%$ & $0 \%$ & $0 \%$ \\
\hline & $\mathrm{C}$ & $0 \%$ & $0 \%$ & $100 \%$ & $0 \%$ & $0 \%$ \\
\hline
\end{tabular}


CHARTS OF RESPONSE AT THE END OF FOLLOW UP RESPONSE OF THE THERAPIES FOR INDIVIDUAL GROUP (PERCENTAGE) AT THE END OF FOLLOW UP

\begin{tabular}{|c|c|c|c|c|c|c|}
\hline Variables & Groups & CD & CS & C1-1 & C1-2 & C1-3 \\
\hline \multirow{3}{*}{ Pain } & $\mathrm{A}$ & $0 \%$ & $70 \%$ & $0 \%$ & $30 \%$ & $0 \%$ \\
\hline & $\mathrm{B}$ & $0 \%$ & $0 \%$ & $40 \%$ & $40 \%$ & $20 \%$ \\
\hline & $\mathrm{C}$ & $0 \%$ & $0 \%$ & $10 \%$ & $60 \%$ & $30 \%$ \\
\hline \multirow{3}{*}{ Bleeding } & $\mathrm{A}$ & $0 \%$ & $10 \%$ & $40 \%$ & $50 \%$ & $0 \%$ \\
\hline & $\mathrm{B}$ & $0 \%$ & $10 \%$ & $40 \%$ & $20 \%$ & $30 \%$ \\
\hline & $\mathrm{C}$ & $0 \%$ & $80 \%$ & $0 \%$ & $10 \%$ & $10 \%$ \\
\hline \multirow{3}{*}{ Nature of Stool } & $\mathrm{A}$ & $0 \%$ & $0 \%$ & $40 \%$ & $60 \%$ & $0 \%$ \\
\hline & $\mathrm{B}$ & $0 \%$ & $90 \%$ & $0 \%$ & $0 \%$ & $0 \%$ \\
\hline & $\mathrm{C}$ & $0 \%$ & $0 \%$ & $10 \%$ & $60 \%$ & $30 \%$ \\
\hline \multirow{3}{*}{ Pressure on defecation } & A & $0 \%$ & $0 \%$ & $40 \%$ & $60 \%$ & $0 \%$ \\
\hline & $\mathrm{B}$ & $0 \%$ & $70 \%$ & $10 \%$ & $20 \%$ & $0 \%$ \\
\hline & $\mathrm{C}$ & $0 \%$ & $10 \%$ & $60 \%$ & $30 \%$ & $0 \%$ \\
\hline \multirow{3}{*}{ Prurituss ani } & $\mathrm{A}$ & $0 \%$ & $0 \%$ & $10 \%$ & $60 \%$ & $30 \%$ \\
\hline & $\mathrm{B}$ & $0 \%$ & $0 \%$ & $60 \%$ & $40 \%$ & $0 \%$ \\
\hline & $\mathrm{C}$ & $0 \%$ & $0 \%$ & $30 \%$ & $40 \%$ & $30 \%$ \\
\hline \multirow{3}{*}{ Mucous Discharge } & $\mathrm{A}$ & $0 \%$ & $0 \%$ & $20 \%$ & $70 \%$ & $10 \%$ \\
\hline & $\mathrm{B}$ & $0 \%$ & $10 \%$ & $70 \%$ & $20 \%$ & $0 \%$ \\
\hline & $\mathrm{C}$ & $0 \%$ & $30 \%$ & $40 \%$ & $30 \%$ & $0 \%$ \\
\hline \multirow{3}{*}{ External } & $\mathrm{A}$ & $0 \%$ & $100 \%$ & $0 \%$ & $0 \%$ & $0 \%$ \\
\hline & $\mathrm{B}$ & $0 \%$ & $100 \%$ & $0 \%$ & $0 \%$ & $0 \%$ \\
\hline & $\mathrm{C}$ & $0 \%$ & $100 \%$ & $0 \%$ & $0 \%$ & $0 \%$ \\
\hline \multirow{3}{*}{ Internal } & A & $0 \%$ & $100 \%$ & $0 \%$ & $0 \%$ & $0 \%$ \\
\hline & $\mathrm{B}$ & $0 \%$ & $100 \%$ & $0 \%$ & $0 \%$ & $0 \%$ \\
\hline & $\mathrm{C}$ & $0 \%$ & $100 \%$ & $0 \%$ & $0 \%$ & $0 \%$ \\
\hline \multirow{3}{*}{ Size } & A & $0 \%$ & $10 \%$ & $50 \%$ & $40 \%$ & $0 \%$ \\
\hline & $\mathrm{B}$ & $0 \%$ & $10 \%$ & $40 \%$ & $50 \%$ & $0 \%$ \\
\hline & $\mathrm{C}$ & $0 \%$ & $0 \%$ & $0 \%$ & $60 \%$ & $40 \%$ \\
\hline \multirow{3}{*}{ Character } & A & $0 \%$ & $30 \%$ & $30 \%$ & $40 \%$ & $0 \%$ \\
\hline & $\mathrm{B}$ & $0 \%$ & $0 \%$ & $40 \%$ & $60 \%$ & $0 \%$ \\
\hline & $\mathrm{C}$ & $0 \%$ & $0 \%$ & $0 \%$ & $70 \%$ & $30 \%$ \\
\hline \multirow{3}{*}{ Pressure on tenderness } & A & $0 \%$ & $60 \%$ & $10 \%$ & $30 \%$ & $0 \%$ \\
\hline & $\mathrm{B}$ & $0 \%$ & $0 \%$ & $40 \%$ & $60 \%$ & $0 \%$ \\
\hline & $\mathrm{C}$ & $0 \%$ & $0 \%$ & $50 \%$ & $50 \%$ & $0 \%$ \\
\hline \multirow{3}{*}{ Movement } & $\mathrm{A}$ & $0 \%$ & $60 \%$ & $0 \%$ & $30 \%$ & $10 \%$ \\
\hline & $\mathrm{B}$ & $0 \%$ & $90 \%$ & $0 \%$ & $10 \%$ & $0 \%$ \\
\hline & $\mathrm{C}$ & $0 \%$ & $90 \%$ & $0 \%$ & $10 \%$ & $0 \%$ \\
\hline \multirow{3}{*}{ Over all } & A & $0 \%$ & $0 \%$ & $10 \%$ & $90 \%$ & $0 \%$ \\
\hline & $\mathrm{B}$ & $0 \%$ & $0 \%$ & $0 \%$ & $100 \%$ & $0 \%$ \\
\hline & $\mathrm{C}$ & $0 \%$ & $0 \%$ & $0 \%$ & $100 \%$ & $0 \%$ \\
\hline
\end{tabular}




\begin{tabular}{|c|c|c|c|c|c|c|c|}
\hline \multicolumn{8}{|c|}{ STATISTICAL ANALYSIS OF GROUP - A } \\
\hline Variables & Grading & Mean & S.D & \begin{tabular}{|l|} 
S.E. \\
\end{tabular} & $\mathbf{t}$ & P. Value & Significance \\
\hline \multirow{3}{*}{ Pain } & B.T & 1.5 & 0.8498 & 0.2687 & - & - & - \\
\hline & A.T & 0.5 & 0.8498 & 0.2687 & 2.63 & 0.169 & NS \\
\hline & F.U & 0.6 & 0.966 & 0.3055 & 2.21 & 0.0401 & $\mathrm{~S}$ \\
\hline \multirow{3}{*}{ Bleeding } & B.T & 3.0 & 0.6667 & 0.2108 & - & - & - \\
\hline & A.T & 1.4 & 0.6992 & 0.2211 & 5.24 & 0.0001 & HS \\
\hline & F.U & 1.4 & 0.6992 & 0.2211 & 5.24 & 0.0001 & HS \\
\hline \multirow{3}{*}{ Nature of Stool } & B.T & 3.3 & 0.6749 & 0.2134 & - & - & - \\
\hline & A.T & 2.2 & 0.7888 & 0.2494 & 3.35 & 0.0036 & $\mathrm{~S}$ \\
\hline & F.U & 1.6 & 0.5164 & 0.1633 & 6.33 & 0.0000 & HS \\
\hline \multirow{3}{*}{ Pressure on defecation } & B.T & 3.3 & 0.6749 & 0.2134 & - & - & - \\
\hline & A.T & 2.2 & 0.7888 & 0.2494 & 3.35 & 0.0036 & $\mathrm{~S}$ \\
\hline & F.U & 1.6 & 0.5164 & 0.1633 & 6.33 & 0.0000 & HS \\
\hline \multirow{3}{*}{ Pruritus ani } & B.T & 3.2 & 0.6325 & 0.2 & - & - & - \\
\hline & A.T & 1.8 & 0.6325 & 0.2 & 4.95 & 0.0001 & HS \\
\hline & F.U & 2.2 & 0.6325 & 0.2 & 3.54 & 0.0024 & $\mathrm{~S}$ \\
\hline \multirow{3}{*}{ Mucous Discharge } & B.T & 3.3 & 0.4830 & 0.1528 & - & - & - \\
\hline & A.T & 1.9 & 0.5676 & 0.1795 & 5.94 & 0.0000 & HS \\
\hline & F.U & 1.9 & 0.5676 & 0.1795 & 5.94 & 0.0000 & $\mathrm{HS}$ \\
\hline \multirow{3}{*}{ External } & B.T & 1.6 & \begin{tabular}{|l|}
1.075 \\
\end{tabular} & \begin{tabular}{|l|}
0.33994 \\
\end{tabular} & - & - & - \\
\hline & A.T & 0.0 & 0.000 & 0.000 & 4.7066 & 0.0001 & HS \\
\hline & F.U & 0.0 & 0.000 & 0.000 & 4.7066 & 0.0001 & HS \\
\hline \multirow{3}{*}{ Internal } & B.T & 1.7 & 0.9487 & 0.29810 & - & - & - \\
\hline & A.T & 0.0 & 0.000 & 0.000 & 5.7026 & 0.0000 & HS \\
\hline & F.U & 0.0 & 0.000 & 0.000 & 5.7026 & 0.0000 & $\mathrm{HS}$ \\
\hline \multirow{3}{*}{ Size } & B.T & 2.5 & 0.5270 & 0.1667 & - & - & - \\
\hline & A.T & 1.1 & 0.8756 & 0.2769 & 4.33 & 0.0004 & HS \\
\hline & F.U & 1.3 & 0.6749 & 0.2134 & 4.43 & 0.0003 & HS \\
\hline \multirow{3}{*}{ Character } & B.T & 2.5 & 0.5270 & 0.1667 & - & - & - \\
\hline & A.T & 1.1 & 0.8756 & 0.2769 & 4.43 & 0.0003 & HS \\
\hline & F.U & 1.1 & 0.8756 & 0.2769 & 4.33 & 0.0004 & $\mathrm{HS}$ \\
\hline \multirow{3}{*}{ Pressure on tenderness } & B.T & 1.9 & 1.287 & 0.4069 & - & - & - \\
\hline & A.T & 0.6 & 0.966 & 0.3055 & 2.55 & 0.0199 & $S$ \\
\hline & F.U & 0.7 & 0.9487 & 0.3 & 2.37 & 0.0289 & $S$ \\
\hline \multirow{3}{*}{ Movement } & B.T & 2.0 & 1.333 & 0.4216 & - & - & - \\
\hline & A.T & 0.9 & 1.197 & 0.3786 & 1.94 & 0.0681 & NS \\
\hline & F.U & 0.9 & 1.197 & 0.3786 & 1.94 & 0.0681 & NS \\
\hline \multirow{3}{*}{ Over all } & B.T & 29.80 & 4.517 & 1.428 & - & - & - \\
\hline & A.T & 16.20 & 1.549 & 0.4899 & 9.01 & 0.0000 & HS \\
\hline & F.U & 15.80 & 2.741 & 0.8667 & 8.38 & 0.0000 & HS \\
\hline
\end{tabular}




\begin{tabular}{|c|c|c|c|c|c|c|c|}
\hline \multicolumn{8}{|c|}{ STATISTICAL ANALYSIS OF GROUP - B } \\
\hline Variables & Grading & Mean & S.D & S.E. & $\mathbf{t}$ & P. Value & Significance \\
\hline \multirow{3}{*}{ Pain } & B.T & 3.000 & 0.6667 & 0.2108 & - & - & - \\
\hline & A.T & 1.900 & 0.7379 & 0.2333 & 3.50 & 0.0026 & $\mathrm{~S}$ \\
\hline & F.U & 1.800 & 0.7888 & 0.2494 & 3.67 & 0.0017 & $\mathrm{~S}$ \\
\hline \multirow{3}{*}{ Bleeding } & B.T & 2.900 & 0.5676 & 0.1759 & - & - & - \\
\hline & A.T & 1.600 & 0.6992 & 0.2211 & 4.56 & 0.0002 & HS \\
\hline & F.U & 1.700 & 1.059 & 0.335 & 3.16 & 0.0054 & $\mathrm{~S}$ \\
\hline \multirow{3}{*}{ Nature of Stool } & B.T & 2.600 & 0.5164 & 0.1633 & - & - & - \\
\hline & A.T & 0.100 & 0.3162 & 0.100 & 13.06 & 0.0000 & $\mathrm{HS}$ \\
\hline & F.U & 0.100 & 0.3162 & 0.100 & 13.06 & 0.0000 & $\mathrm{HS}$ \\
\hline \multirow{3}{*}{ Pressure on defecation } & B.T & 2.800 & 0.4216 & 0.133 & - & - & - \\
\hline & A.T & 0.100 & 0.3162 & 0.100 & 16.20 & 0.0000 & $\mathrm{HS}$ \\
\hline & F.U & 0.500 & 0.8492 & 0.2687 & 7.67 & 0.0000 & $\mathrm{HS}$ \\
\hline \multirow{3}{*}{ Pruritus ani } & B.T & 2.500 & 0.5270 & 0.1667 & - & - & - \\
\hline & A.T & 1.400 & 0.5164 & 0.1663 & 4.71 & 0.0002 & HS \\
\hline & F.U & 1.400 & 0.5164 & 0.1663 & 4.71 & 0.0002 & $\mathrm{HS}$ \\
\hline \multirow{3}{*}{ Mucous Discharge } & B.T & 2.500 & 0.5270 & 0.1667 & - & - & - \\
\hline & A.T & 1.400 & 0.5164 & 0.1663 & 4.71 & 0.0002 & $\mathrm{HS}$ \\
\hline & F.U & 1.100 & 0.5676 & 0.1795 & 5.72 & 0.0000 & $\mathrm{HS}$ \\
\hline \multirow{3}{*}{ External } & B.T & 2.3 & 0.8233 & 0.2603 & - & - & - \\
\hline & A.T & 0.0 & 0.0000 & 0.0000 & 8.834 & 0.0000 & HS \\
\hline & F.U & 0.0 & 0.0000 & 0.0000 & 8.834 & 0.0000 & HS \\
\hline \multirow{3}{*}{ Internal } & B.T & 1.1 & 0.3162 & 0.0999 & - & - & - \\
\hline & A.T & 0.0 & 0.0000 & 0.0000 & 11.011 & 0.0000 & $\mathrm{HS}$ \\
\hline & F.U & 0.0 & 0.0000 & 0.0000 & 11.011 & 0.0000 & HS \\
\hline \multirow{3}{*}{ Size } & B.T & 2.800 & 0.6325 & 0.200 & - & - & - \\
\hline & A.T & 1.600 & 0.5164 & 0.1633 & 4.65 & 0.0002 & HS \\
\hline & F.U & 1.600 & 0.5164 & 0.1633 & 4.65 & 0.0002 & HS \\
\hline \multirow{3}{*}{ Character } & B.T & 2.700 & 0.6749 & 0.2134 & - & - & - \\
\hline & A.T & 1.400 & 0.6992 & 0.2211 & 4.23 & 0.0005 & HS \\
\hline & F.U & 1.400 & 0.6992 & 0.2211 & 4.23 & 0.0005 & HS \\
\hline \multirow{3}{*}{ Pressure on tenderness } & B.T & 2.700 & 0.4830 & 0.1528 & - & - & - \\
\hline & A.T & 1.400 & 0.5164 & 0.1633 & 5.81 & 0.0000 & $\mathrm{HS}$ \\
\hline & F.U & 1.600 & 0.5164 & 0.1633 & 4.92 & 0.0001 & HS \\
\hline \multirow{3}{*}{ Movement } & B.T & 1.200 & 0.6325 & \begin{tabular}{|l|}
0.200 \\
\end{tabular} & - & - & - \\
\hline & A.T & 0.200 & 0.6325 & 0.200 & 3.54 & 0.0024 & $\mathrm{~S}$ \\
\hline & F.U & 0.200 & 0.6325 & \begin{tabular}{|l|}
0.200 \\
\end{tabular} & 3.54 & 0.0024 & $S$ \\
\hline \multirow{3}{*}{ Over all } & B.T & 29.00 & 2.055 & 0.6498 & - & - & - \\
\hline & A.T & 17.90 & 1.524 & 0.4819 & 13.72 & 0.0000 & HS \\
\hline & F.U & $\mid 17.50$ & 1.958 & 0.6191 & 12.81 & 0.0000 & HS \\
\hline
\end{tabular}




\begin{tabular}{|c|c|c|c|c|c|c|c|}
\hline \multicolumn{8}{|c|}{ STATISTICAL ANALYSIS OF GROUP - C RESULTS } \\
\hline Variables & Grading & Mean & S.D & S.E. & $\mathbf{t}$ & P. Value & Significance \\
\hline \multirow{3}{*}{ Pain } & B.T & 3.3 & 0.4830 & 0.1528 & - & - & - \\
\hline & A.T & 2.2 & 0.6325 & 0.200 & 4.37 & 0.0004 & HS \\
\hline & F.U & 2.2 & 0.6325 & 0.200 & 4.37 & 0.0004 & HS \\
\hline \multirow{3}{*}{ Bleeding } & B.T & 1.5 & 1.080 & 0.3416 & - & - & - \\
\hline & A.T & 0.5 & 1.080 & 0.3416 & 2.07 & 0.0531 & NS \\
\hline & F.U & 0.5 & 1.080 & 0.3416 & 2.07 & 0.0531 & NS \\
\hline \multirow{3}{*}{ Nature of Stool } & B.T & 3.3 & 0.483 & 0.1528 & - & - & - \\
\hline & A.T & 2.2 & 0.6325 & 0.200 & 4.37 & 0.0004 & HS \\
\hline & F.U & 2.2 & 0.6325 & 0.200 & 4.37 & 0.0004 & HS \\
\hline \multirow{3}{*}{ Pressure on defecation } & B.T & 3.3 & 0.4830 & 0.1528 & - & - & - \\
\hline & A.T & 2.2 & 0.6325 & 0.2 & 4.37 & 0.0004 & HS \\
\hline & F.U & 2.2 & 0.6325 & 0.2 & 4.37 & 0.0004 & HS \\
\hline \multirow{3}{*}{ Pruritus ani } & B.T & 3.1 & 0.7379 & 0.233 & - & - & - \\
\hline & A.T & 2.0 & 0.8165 & 0.2582 & 3.16 & 0.0054 & $\mathrm{~S}$ \\
\hline & F.U & 2.0 & 0.8165 & 0.2582 & 3.16 & 0.0054 & $\mathrm{~S}$ \\
\hline \multirow{3}{*}{ Mucous Discharge } & B.T & 3.1 & 0.7379 & 0.2333 & - & - & - \\
\hline & A.T & 2.0 & 0.8165 & 0.2582 & 3.16 & 0.0054 & $S$ \\
\hline & F.U & 2.0 & 0.8165 & 0.2582 & 3.16 & 0.0054 & $S$ \\
\hline \multirow{3}{*}{ External } & B.T & 2.4 & 0.8433 & 0.26667 & - & - & - \\
\hline & A.T & 0.0 & 0.0000 & 0.000 & 8.9997 & 0.0000 & HS \\
\hline & F.U & 0.0 & 0.0000 & 0.000 & 8.9997 & 0.0000 & HS \\
\hline \multirow{3}{*}{ Internal } & B.T & 1.1 & 0.3162 & 0.09999 & - & - & - \\
\hline & A.T & 0.0 & 0.0000 & 0.0000 & 11.010 & 0.0000 & HS \\
\hline & F.U & 0.0 & 0.0000 & 0.0000 & 11.010 & 0.0000 & HS \\
\hline \multirow{3}{*}{ Size } & B.T & 3.5 & 0.5270 & 0.1667 & - & - & - \\
\hline & A.T & 2.4 & 0.5164 & 0.1633 & 4.71 & 0.0002 & HS \\
\hline & F.U & 2.4 & 0.5164 & 0.1633 & 4.71 & 0.0002 & HS \\
\hline \multirow{3}{*}{ Character } & B.T & 3.5 & 0.5270 & 0.1667 & - & - & - \\
\hline & A.T & 2.3 & 0.4830 & 0.1528 & 5.31 & 0.0000 & HS \\
\hline & F.U & 2.3 & 0.4830 & 0.1528 & 5.31 & 0.0000 & $\mathrm{HS}$ \\
\hline \multirow{3}{*}{ Pressure on tenderness } & B.T & 2.6 & 0.5164 & 0.1633 & - & - & - \\
\hline & A.T & 1.5 & 0.5270 & 0.1667 & 4.71 & 0.0002 & HS \\
\hline & F.U & 1.5 & 0.5270 & 0.1667 & 4.71 & 0.0002 & HS \\
\hline \multirow{3}{*}{ Movement } & B.T & 1.2 & 0.6325 & 0.2 & - & - & - \\
\hline & A.T & 0.2 & 0.6325 & 0.2 & 3.54 & 0.0024 & $\mathrm{~S}$ \\
\hline & F.U & 0.2 & 0.6325 & 0.2 & 3.54 & 0.0024 & $S$ \\
\hline \multirow{3}{*}{ Over all } & B.T & 31.90 & 2.132 & 0.6741 & - & - & - \\
\hline & A.T & 14.40 & 1.075 & 0.3399 & 23.18 & 0.0000 & HS \\
\hline & F.U & 14.40 & 1.075 & 0.3399 & 23.18 & 0.0000 & HS \\
\hline
\end{tabular}




\section{Disscussion}

On title

However modern science as advanced and reached highest goal in medical aspects. It is yet to come up with complete treatment plan for arsha as surgical procedures find unjudicial application. The present study tittled "The Medical Management of Arsha with chirabilwadi quatha and kaseesadi thaila sthanika abhyanga" is a sincere attempt for the management of arsha which could be practicable at O.P.D. levels too.

\section{Probable mode of action}

\section{Chirabilwadi quatha}

Here, due to nidanakara factors apanavata gets aggravated that afflicts the anal sphincter leads to arsha. The ingredients of chirabilwadi quatha is having vata shamana properties. So it decreases the aggravated apanavata and prevents the affliction to anal sphincters and cures the arsha.

\section{Kaseesadi Taila}

It acts on haemorrhoids in two ways:

1. It cauterises the pile mass directly because of its ksharana guna (corrosive nature) and makes ropana (healing) of the same.

2. It coagulates protein in haemorrhoidal plexus.

The coagulation of protein leads to disintegration of haemoglobin into haem and globin. Synergy of these action result in decreasing the size of the pile mass. Further, necrosis of the tissue in the haemorrhoidal vein will occur. The necrosed tissue slough out as blackish brown. The haem present in the slough gives the discharge its colour. The tissue becomes fibrosed and scar formation seen. The haemorrhoidal vein obliterates permanently and there is no recurrence of haemorrhoids.

\section{On Results}

All three groups had shown high significant at the end of treatment and all three groups were withheld to end of treatment and end of follow up.

\section{Conclusions}

1) Majority chirabilwadi quatha relieves the subjective criteria variables and kaseesadi thaila relieves the objective criteria variables.

2) Observed that reduction of varicosed haemorroidal veins.

3) All the groups showed highly significant after treatment and after follow up as compared to before treatment (on the basis of $\mathrm{P}$ value). Whereas group $\mathrm{C}$ shows high significant difference as compare with group A and group B. (on the basis of ' $t$ ' value).

4) In the overall clinical study effective medical management is possible but not complete cure.

\section{References}

1. Shastri R, editor. Harita Samhita (text with Asha Hindi commentary) of Harita. Arsha chikitsa. $1^{\text {st }}$ edn., Varanasi: Pracha prakashana, 1985: (Tritiya sthana): 264-276.

2. Thomson W H, Oxford Text book of Surgery. $2^{\text {nd }}$ edn., New York: Oxford University press, 2000: 1551-1568.

3. Nelson H, Dozois R R, Sabiston- text book of surgery. $16^{\text {th }}$ edn., Singarpore: Harcourt PTE Ltd, 2001: 974-996.

4. Yadavaji T A, editor. Susrhuta samhita (with the Nibandhasangraha commentary of Shri Dalhanacharya and the Nyayachandrika Panjika of Shri Gayadasacharya on Nidanasthana). Arsha Nidana. $7^{\text {th }}$ edn., Varanasi: Chaukhamba orientalia, 2002: (Nidanasthana): 270-276.

5. Anderson K N, Mosby's Medical, Nursing and Allied Health. $4^{\text {th }}$ edn., 
London: St. Louis Baltimare Baston, 2000: 728.

6. Sharma S, editor. Sahasrayogam (mula sanskrit evam malayalam, Hindi, Anuvada sahit) of Sharma R. $2^{\text {nd }}$ edn., Hydrabad: Dakshin prakashan, 1990: 17.

7. Mishra B S, editor. Rasendrasara Sangraha (Rasachandrika Hindi commentary) of Krsna G. $6^{\text {th }}$ edn., Varanasi: Chaukhamba Amarabharati prakashan, 1981: 206.

8. Govt. of India Ministry of health and family welfare Dept. of I.S.M and H. The Ayurvedic pharamacopocia of India. New Delhi: $1^{\text {st }}$ edn., 2 (pt 1): 133. 